\title{
Kommentar II zum Fall: „Mutmaßlicher Widerruf einer Patientenverfügung?"“
}

\author{
Gunnar Duttge $\cdot$ Mirjam Schander
}

Online publiziert: 15 . September 2010

(C) Die Autor(en) 2010. Dieser Artikel ist auf Springerlink.com mit Open Access verfügbar.

Vor dem Hintergrund einer verbreiteten Sorge der Menschen, in den Händen der Intensivmedizin „entmündigt“ zu werden, hat das neue „Patientenverfügungsgesetz“ (in Kraft getreten am 1.9.2009) erklärtermaßen zum Ziel, dem Selbstbestimmungsrecht der Patienten größtmögliche Geltung zu verschaffen. Dieses Anliegen soll auch und gerade dann Wirkung entfalten, wenn die Betroffenen infolge ihrer (jedenfalls rechtlichen) Handlungsunfähigkeit selbst nicht mehr sicherstellen können, dass ihr von ärztlicher Vernunft evtl. abweichender Wille vorbehaltlos respektiert wird. Wie sehr Menschen daran bisher Zweifel hegen mussten, zeigt das vorliegende Fallgeschehen in aller Deutlichkeit:

Schon bei der Notfallaufnahme wird den behandelnden Ärzten bekannt, dass der mutmaßliche Wille des eingelieferten Patienten einer künstlichen Beatmung womöglich ,entschieden“ zuwiderläuft. Dass dabei eine höchstpersönliche Grundhaltung dieses Patienten in Frage stehen könnte, legt schon die Krankengeschichte nahe und bestätigt sich im weiteren Verlauf dadurch, dass die nichtinvasive Maskenbeatmung trotz fortlaufender Nachfragen beharrlich abgelehnt wird. Nur: Die Medizin sorgt dafür, dass der Patient sich selbst nicht mehr durchgehend zur Wehr setzen kann: Von wenigen abendlichen Stunden in stabilerem Zustand abgesehen, wird er mehrfach sediert und zunächst - entgegen einer Absprache mit der Tochter - erneut der Maskenbeatmung unterworfen und schließlich zur operativen Behebung des hierdurch erst entstandenen zusätzlichen Leidens tracheotomiert und bronchoskopiert.

Die Möglichkeiten, seinem am Abend sogar ausdrücklich bekundeten Willen Rechnung zu tragen, sind durch ein Zusammenwirken der Beteiligten geradezu systematisch ignoriert worden: Bereits zu Beginn wurde das Vorhandensein einer Patientenverfügung mit eben jenem ablehnenden Willen bekannt, ohne dass es Angehörige wie behandelnde Ärzte für nötig hielten, die zeitnahe Kenntnisnahme des konkreten Inhalts sicherzustellen und nicht dem Belieben der Ehefrau zu überlassen. Mit der unmissverständlichen und wiederholten Willensäußerung am Abend in einem offenbar allgemein als einwilligungsfähig betrachteten Zustand gab es keinen Zweifel mehr über den Inhalt des Patientenwillens und keinen

G. Duttge $(\bowtie) \cdot M$. Schander

Zentrum für Medizinrecht, Georg-August-Universität Göttingen,

Goßlerstr. 19, 37073 Göttingen, Deutschland

E-Mail: lduttge@gwdg.de 
Interpretationsspielraum. Die am Folgetag vorgelegte Patientenverfügung hatte daher juristisch an Bedeutung gänzlich verloren: Hätte ihr Inhalt in Widerspruch zum vorabendlich Bekundeten gestanden, so wäre darin ein (formlos wirksamer, §1901a Abs. 1 S.3 BGB) Widerruf des zuvor Vorausverfügten zu sehen gewesen. Der aktuell erklärte Wille ist, soweit in einwilligungsfähigem Zustand kundgetan, einer Patientenverfügung stets vorrangig. Hier beanspruchte der eindeutige Patientenwille aber umso mehr Beachtung, als er sich mit dem Inhalt der Patientenverfügung offenbar nahtlos deckte. Die von der Ehefrau vorgenommene „Interpretation“, mit der die ablehnende Haltung unter den Vorbehalt eines besseren Gesundheitszustandes gestellt wurde, stand daher schon deshalb in dringendem Verdacht, den wirklichen Patientenwillen zu verfehlen. Zudem widersprach diese Deutung eklatant dem tatsächlichen Geschehen am Vorabend, als der Patient trotz verbessertem Zustand die Maskenbeatmung weiterhin dediziert ablehnte.

Überdies beanspruchte die Ehefrau eine Befugnis, die ihr von Rechts wegen gar nicht zustand: Trotz dahingehenden Vermerkes in der Patientenverfügung kann sie als Gesundheitsbevollmächtigte nur dann wirksam auftreten, wenn sie nachweist, dass ihr die Sorge in Gesundheitsangelegenheiten unter ausdrücklicher Nennung des Rechts zur Einwilligung in Heilbehandlungen und ärztliche Eingriffe schriftlich überantwortet wurde; eine bloß abstrakt formulierte Generalvollmacht genügt nicht (vgl. § 1904 Abs. 5 S. 2 BGB). Da das deutsche Recht eine „,natürliche Stellvertretung“ kraft familiärer Verbundenheit nicht kennt, war ihr Verlangen auf Wiederaufnahme der Beatmung unbeachtlich. Ohne die vorabendlichen Vorgaben des Patienten wäre in einem solchen Fall zwecks Herstellung seiner juristischen Handlungsfähigkeit ohne Zeitverzug eine Betreuerbestellung zu veranlassen gewesen; das hierfür zuständige Betreuungsgericht hätte ggf. auch die Rolle der Ehefrau und deren Interpretation klarstellen können. Aufgrund der ausdrücklichen Patientenerklärung gab es jedoch nur eine richtige Handlungsmaxime: diesen Willen - notfalls auch unter den Protesten der Angehörigen - zu befolgen.

Der Eindruck einer somit krassen Missachtung des Selbstbestimmungsrechts (auch bzgl. der OP, für die es ebenfalls an einer wirksamen Einwilligung fehlte) wird schließlich weder durch ein nachträgliches Schweigen - ja selbst durch ein nachträgliches Gutheißen - des Patienten noch dadurch in Frage gestellt, dass er zumindest vor der Tracheotomie den Blickkontakt (wirklich zum behandelnden Arzt?) aufnahm: Die Annahme eines Widerrufs der vorausgehenden Entscheidung setzt mindestens Gesten oder nonverbales Verhalten von solcher Art voraus, dass auf einen solchen Erklärungswert hinreichend verlässlich geschlossen werden kann. Manche verlangen sogar eine ausdrückliche (verbale) Erklärung oder das Bestehen von Einwilligungsfähigkeit. Der bloße Blickkontakt ohne vorausgehende Frage hat keinerlei fassbaren Erklärungsinhalt und gibt allenfalls Raum für Spekulationen. Vor solchen soll der Patient jedoch durch das neue Gesetz geschützt werden: Ein nur ,,mutmaßlicher Widerruf" ist eben keiner!

Open Access Dieser Artikel unterliegt den Bedingungen der Creative Commons Attribution Noncommercial License. Dadurch sind die nichtkommerzielle Nutzung, Verteilung und Reproduktion erlaubt, sofern der/die Originalautor/en und die Quelle angegeben sind. 